\title{
Numerical Characterization of Vanadium Dioxide Thin Films Applied to Thermal Building Insulation: Determination of Dielectric Constant Using PSO Algorithm
}

\author{
Omar Iken ${ }^{\# 1}$, Hamid Bouyghf*, Kenza Bouazaoui", Rachid Agounoun ${ }^{\#}$, Miloud Rahmoune ${ }^{\#}$, Amine \\ Zoubir $^{\#}$, Khalid Sbai ${ }^{\#}$, Rachid Saadani ${ }^{\#}$, Abdelali Rahmani* ${ }^{* *}$ \\ " Laboratoire d'Etude des Matériaux Avancés et Applications (LEM2A), Université Moulay Ismail, \\ Faculté des sciences, Ecole Supérieure de Technologie Meknès, Morocco \\ 1 omar.iken0@gmail.com \\ *LSSDIA, ENSET Mohammedia, University of Hassan II Casablanca, BP 159 Bd Hassan II, \\ Mohammedia-Morocco. \\ ** Laboratoire d'Etude des Matériaux Avancés et Applications (LEM2A), Université Moulay Ismail, \\ Faculté des sciences, Meknès, Morocco
}

\begin{abstract}
Radiative rectification is one of the newest solutions for thermal building insulation. It is a phenomenon which has good analogy with electronic diode principle. It consists on being blocking for solar radiations in summer and passing radiations in winter. Among materials which have rectification capacity, Vanadium dioxide $\left(\mathrm{VO}_{2}\right)$ offers good applicability to building insulation with quite good rectification efficiency. $\mathrm{VO}_{2}$ has a transition temperature around $68^{\circ} \mathrm{C}$. Under this temperature, the material has a semiconductor crystallographic structure; therefore it becomes transparent to visible and infrared solar spectra. Above $68^{\circ} \mathrm{C}$, the crystallographic structure of $\mathrm{VO}_{2}$ changes to a metallic state for which it becomes more reflective to the same spectra. This semiconductor/metallic transition influences the optical properties of $\mathrm{VO}_{2}$ which are highly dependent to temperature and wavelength. These properties are refractive index $n(T, \lambda)$ and extinction coefficient $k(T, \lambda)$, they are necessary in the optical study of $\mathrm{VO}_{2}$ insulation capacities. Determination of these properties is purely experimental using ellipsometry techniques. In this work we suggest a method for numerical determination of these optical properties. This method uses the Particle Swarm Optimization (PSO) algorithm and it is based on the theoretical model of Lorentz oscillators at $\mathrm{VO}_{2}$ nanoparticles scale. We calculated $\mathrm{n}(\mathrm{T}, \lambda)$ and $\mathrm{k}(\mathrm{T}, \lambda)$ for 3 temperatures and compared them with experimental results. This comparison showed good agreement between numerical and experimental results.
\end{abstract}

Vanadium-dioxide, radiative rectification, building insulation, optical properties, optimization algorithms.

\section{INTRODUCTION}

Vanadium dioxide is a metal oxide that attracts many research teams over the world. It offers thermo-chromic properties depending on its semiconductor/metallic phase transition around $68^{\circ} \mathrm{C}[1,2] . \mathrm{VO}_{2}$ has many potential applications as a photonic device. In our case, we are trying to use $\mathrm{VO}_{2}$ as a smart solution for radiative rectification applied to thermal building insulation, a smart solution because of its thermo-chromic properties highly dependent to temperature. At temperatures less than $68^{\circ} \mathrm{C}, \mathrm{VO}_{2}$ has a semiconductor crystallographic structure, with inter-atomic distances which allow high radiative transmission. Above $68^{\circ} \mathrm{C}, \mathrm{VO}_{2}$ switches to a metallic structure with small inter-atomic distances, thus $\mathrm{VO}_{2}$ become more reflective to solar radiations. $\mathrm{VO}_{2}$ is proposed for application to building glazing as smart window coatings by C.G. Granqvist et al [3]. The transition temperature too high compared with the comfort temperature is one of the limitations for smart windows application. G.V. Jorgenson et al [4] and W. Burkhardt et al [5] proposed a solution to decrease the $\mathrm{VO}_{2}$ transition temperature by doping with some materials such as tungsten (W), Fluoride (F) and Molybdenum (Mo) with calculated concentrations to adjust the transition temperature around $25^{\circ} \mathrm{C}$. The second limitation is related to high absorption in the visible part of solar spectrum, this phenomenon was noticed experimentally by C.G. Granqvist et al [6] and Y. Gao et al [7], and numerically O. Iken et al [8] using the kubelka Munk method.

The study of thermo-chromic properties of $\mathrm{VO}_{2}$ requires data of optical constants (refractive index $\mathrm{n}(\mathrm{T}, \lambda)$ and extinction coefficient $\mathrm{k}(\mathrm{T}, \lambda)$ ) for different temperatures above and under transition. The determination of those two properties is purely experimental, thus it requires simple fabrication and characterization by ellipsometry [9]. This process is expensive and the equipments are not always available, therefore, a numerical alternative could be a good solution to acquire optical constants. 
In this paper, we present a numerical method to found $\mathrm{VO}_{2}$ optical constants. This method uses the particle swarm optimization (PSO) algorithm [10] which is inspired from the social behavior of birds flocking in their food finding process. A bird flock does not have any leader, therefore, birds follow one of the members of the group that have the closest position with a food source (potential solution), this position will be transmitted to other members in the flock and a convergence phenomena occurs to optimize the food source position. This intelligent algorithm begin from a random point in the search space and uses its memory to record its best position, every bird for example will remember the global best position for all the flock (Global Best) and also his individual best position (Local Best). Those two positions change dynamically depending on the velocity of the population which is one of the most important parameters for the algorithm convergence. The PSO algorithm like other optimization algorithms $[11,12]$ needs an objective function with parameters to optimize. In our case, the objective function is the equation describing the theoretical model of Lorentz oscillators. This model calculates the dielectric constant as a function of photon energy by the sum of three Lorentz oscillators which describe interbands transition between energy levels of Oxygen and Vanadium atoms [13].

The objective of this work is to estimate the optical constants of $\mathrm{VO}_{2}$ just by having an idea about the variation interval of the 3 Lorentz oscillators' parameters. The PSO algorithm uses this interval as the search space and tries to found the optimal parameters combination that gives the minimum of the objective function. Using this method, we estimate numerically the refractive index and the extinction coefficient values for 3 temperatures around the $\mathrm{VO}_{2}$ metal /semiconductor transition. A comparison with experimental results reported by $\mathrm{H}$. Kakiuchida et al $[13,14]$ is done.

The next section presents the theoretical model used in this work and also some details about the PSO algorithm. In the results section, we expose our findings with discussion and comparison. We conclude with a results recapitulation and perspectives.

\section{THEORY}

Lorentz oscillators' model was computed as an objective function for the PSO algorithm in the aim of estimating numerically the dielectric constant of $\mathrm{VO}_{2}$ and then extract refractive index $\mathrm{n}(\mathrm{T}, \lambda)$ (real part of the dielectric constant) and extinction coefficient $\mathrm{k}(\mathrm{T}, \lambda)$ (imaginary part of the dielectric constant).

\section{A. Theoretical model of Lorentz oscillators}

Dielectric constant or permittivity expresses the atoms behaviour in a material when electric field is applied [15]. This behaviour was modelled by Lorentz as the interaction of the electric field with the charge of the electron which is considered as a mass bounded to the nucleus of the atoms by a spring, thus this interaction causes stretching and compression of the spring, which would set the electron into oscillating motion. These oscillations are related to the electronic configuration and the densities of states at the atomic orbital level. Every free carrier in the peripheral bands inside an atom is considered as an oscillator and can be described by its amplitude $\mathrm{Am}_{\mathrm{j}}$, energy $\mathrm{E}_{\mathrm{j}}$ and its dumping coefficient $\mathrm{Br}_{\mathrm{j}}$ with $\mathrm{j}$ the index of the oscillator. 14].

In our case, vanadium dioxide $\mathrm{VO}_{2}$ dielectric constant can be written as a sum of three oscillators $(\mathrm{j}=3)[13$,

$$
(n(\lambda, T)+i k(\lambda, T))^{2}=\varepsilon_{\infty}+\sum_{j=1}^{3} \frac{A m_{j}}{E_{j}^{2}-E_{p}^{2}-i B r_{j} E_{p}}
$$

With $E_{p}=h c / \lambda$

$E_{\mathrm{P}}$ is the photon energy of the incident radiations with $\mathrm{c}$ the speed of light, $\mathrm{h}$ the Planck constant and $\lambda$ the wavelength, $\varepsilon_{\infty}$ is the dielectric constant at $E_{p}=\infty$. The first oscillator $(\mathrm{j}=1)$ is related to the interband transition from the oxygen (O) $2 p$ band to the empty vanadium $(\mathrm{V}) 3 \mathrm{~d}$ band, it corresponds to the reduction of the energy gap between the $2 p$ band of oxygen and the vanadium empty $3 d$ band lowered down to the Fermi level. The second oscillator $(\mathrm{j}=2)$ is due to the transition of trapped carriers between split $3 \mathrm{~d}$ bands, it is the primary factor of the weak broad absorption in the near-infrared region according to H. Kakiuchida et al [13]. The third oscillator $(\mathrm{j}=3)$ is attributed to the existence of another interband transition at the metallic phase.

Equation (1) is used as the objective function of the PSO algorithm with the oscillator's amplitude, energy and dumping coefficient as variables to be optimized.

\section{B. Particle Swarm Optimization algorithm}

The particle swarm is an algorithm for finding optimal regions of complex search spaces through the interaction of individuals in a population of particles according to M. Clerc et al [10]. The individual particles are attracted stochastically toward the positions of their own best fitness achieved so far and the best fitness achieved so far by any of their neighbours. The particles population is initialized with random positions and velocities and an objective function is evaluated using the particle's positional coordinates as input values. In 
this work, those coordinates are represented by the values of the Lorentz oscillators parameters $\left(\mathrm{Am}_{\mathrm{j}}, \mathrm{Ej}_{\mathrm{j}} \mathrm{Br}_{\mathrm{j}}\right.$ and $\varepsilon_{\infty}$ ) and the search space is built by their variation intervals (low (l) and high (h) bounds) as vectors (tab.1). The optimization result is a vector which belongs to the search space presented by the Matrix (2) and verifies the local minimum -for every value of the photon energy (wavelength) - of the objective function.

$$
\begin{array}{cccccccccc}
\varepsilon_{\infty l} & A m_{1 l} & E_{1 l} & B r_{1 l} & A m_{2 l} & E_{2 l} & B r_{2 l} & A m_{3 l} & E_{3 l} & B r_{3 l} \\
\vdots & \vdots & \vdots & \vdots & \vdots & \vdots & \vdots & \vdots & \vdots & \vdots \\
\vdots & \vdots & \vdots & \vdots & \vdots & \vdots & \vdots & \vdots & \vdots & \vdots \\
\vdots & \vdots & \vdots & \vdots & \vdots & \vdots & \vdots & \vdots & \vdots & \vdots \\
\varepsilon_{\infty h} & A m_{1 h} & E_{1 h} & B r_{1 h} & A m_{2 h} & E_{2 h} & B r_{2 h} & A m_{3 h} & E_{3 h} & B r_{3 h}
\end{array}
$$

\begin{tabular}{|c|c|c|c|c|c|c|c|c|c|c|c|}
\hline \multicolumn{2}{|c|}{ Parameters } & $\varepsilon_{\infty}$ & $\mathbf{A m}_{1}$ & $\mathbf{E}_{1}$ & $\mathrm{Br}_{1}$ & $\mathrm{Am}_{2}$ & $\mathbf{E}_{2}$ & $\mathrm{Br}_{2}$ & $\mathbf{A m}_{3}$ & $\mathbf{E}_{3}$ & $\mathrm{Br}_{3}$ \\
\hline \multirow{2}{*}{$\begin{array}{c}\mathrm{T}< \\
68^{\circ} \mathrm{C} \\
\end{array}$} & Low & 3 & 42 & 3.5 & 1 & 4 & 1.35 & 1 & 1 & 1 & 1 \\
\hline & High & 5.5 & 44 & 3.6 & 1.4 & 5 & 1.36 & 1.4 & 1 & 1 & 1 \\
\hline \multirow{2}{*}{$\begin{array}{c}T= \\
68^{\circ} \mathrm{C}\end{array}$} & Low & 3 & 30 & 3.2 & 0.6 & 9 & 0.5 & 1.3 & 1 & 0.5 & 0.5 \\
\hline & High & 5.5 & 35 & 3.3 & 0.9 & 14 & 0.9 & 1.5 & 2 & 2 & 0.7 \\
\hline \multirow{2}{*}{$\begin{array}{c}T> \\
68^{\circ} \mathrm{C}\end{array}$} & Low & 4.8 & 23 & 3.1 & 0.7 & 16 & 0.5 & 0.7 & 1 & 2.5 & 0.5 \\
\hline & High & 5.2 & 25 & 3.3 & 1 & 18 & 1 & 1 & 2 & 2.6 & 0.7 \\
\hline
\end{tabular}

Table 1. Lorentz oscillator's parameters bounds

The values presented in (tab.1) are approximated using experimental curves presented by Kakiuchida et al [13]. Those curves describe the evolution of Lorentz oscillator's parameters as function of temperature.

This search process is repeated several times (20 runs) to be sure of the reproducibility of the optimization results, every run spends around 34 seconds. The particles population used in this optimization is composed of 100 individuals with 100 generations. Our objective function is a complex equation, thus the fitness of the optimization solutions is also complex. The PSO convergence characteristics for this application are presented in (fig.1, 2).

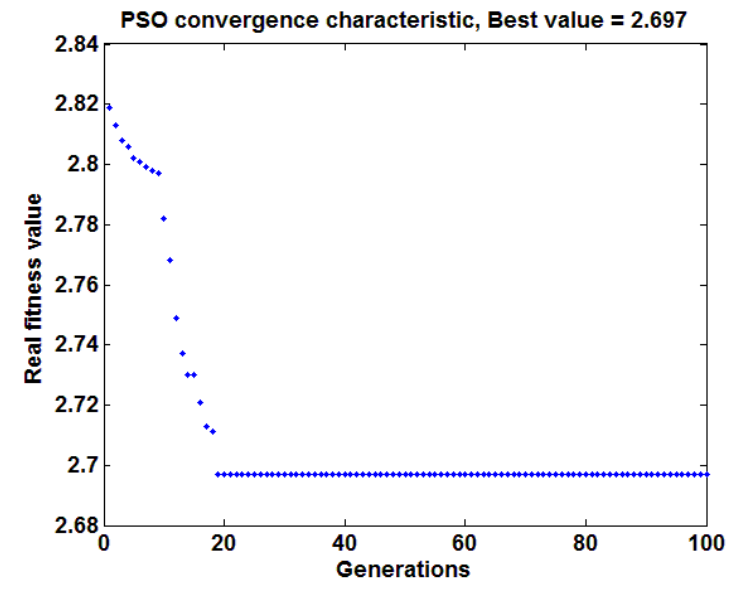

Fig. 1. Fitness convergence of the real part (refractive index) versus generations at $\lambda=2000 \mathrm{~nm}$

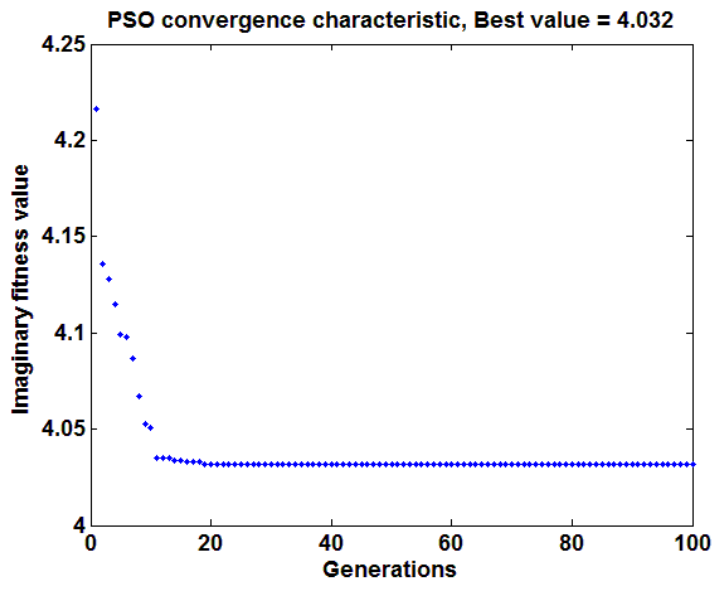

Fig. 2. Fitness convergence of the imaginary part (extinction coefficient) versus generations at $\lambda=2000 \mathrm{~nm}$

The PSO convergence characteristics show a good convergence for the two parts (real and imaginary) of the fitness function. After 100 generations, the best fitness values stay close to 2.697 for the refractive index and 4.032 for the extinction coefficient at a wavelengths around $\lambda=2000 \mathrm{~nm}$ for $\mathrm{T}>68^{\circ} \mathrm{C}\left(\mathrm{VO}_{2}\right.$ at metallic state). Almost after 19 generations, the fitness values remain constant and increasing more generations does not decrease the best values.

\section{III.RESULTS AND DISCUSSION}

We used the Particles swarm optimization algorithm to estimate the optical properties (refractive index $\mathrm{n}$ and extinction coefficient $\mathrm{k}$ ) of $\mathrm{VO}_{2}$ at the semiconductor state $\left(<68^{\circ} \mathrm{C}\right)$, at the transition $\left(=68^{\circ} \mathrm{C}\right)$ and at the metallic state $\left(>68^{\circ} \mathrm{C}\right)$. The theoretical model of Lorentz oscillators (eq.1) was used as the objective function to be optimized by the PSO algorithm. This model calculates directly the dielectric constant by optimizing its 10 variables initialized by lower and upper bounds (tab. 1). This method of optimization gives us some interesting 
results (fig.3, 4, 5), compared to the experimental results given by Kakiuchida et al [13, 14] and C.G. Granqvist et al [6], our results are in good agreement and show a high similarity with an average error of about 7,5\% with experimental ones.

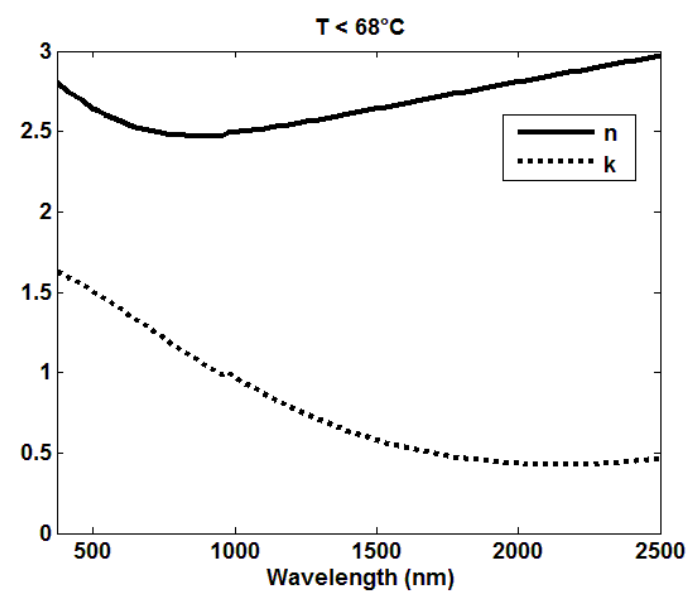

Fig. 3. Refractive index and extinction coefficient versus wavelength at semiconductor state.

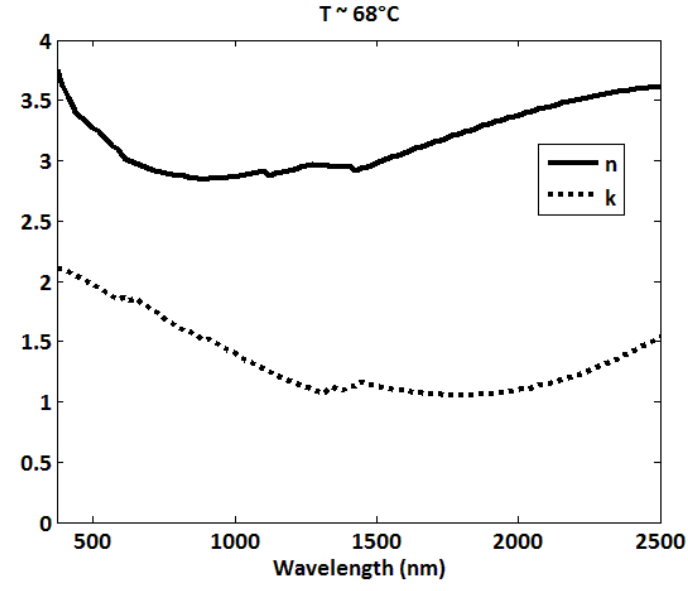

Fig. 4. Refractive index and extinction coefficient versus wavelength at transition.

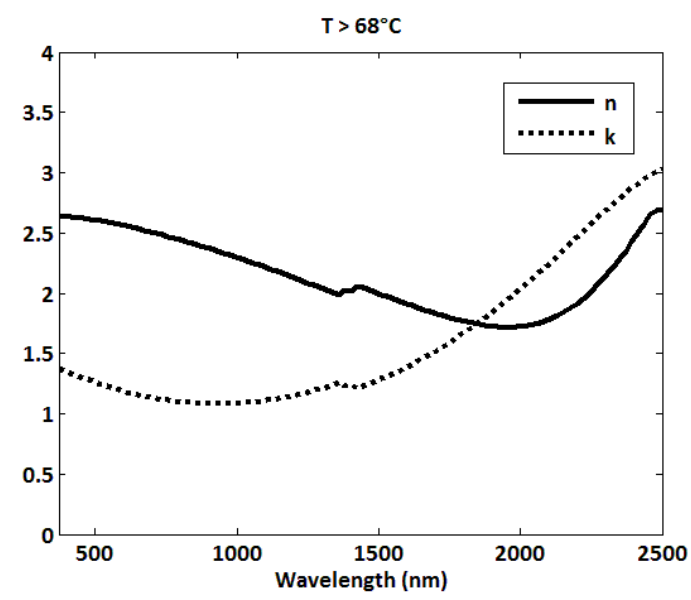

Fig. 5. Refractive index and extinction coefficient versus wavelength at metallic state.

We can observe in figure 4 and 5 some distortions in the curves near $1500 \mathrm{~nm}$. Those distortions are due to some minor errors during the convergence process of the algorithm. As a result, we obtain some shifted points on the $\mathrm{n}$ and $\mathrm{k}$ curves, spatially in fig. 4 because at the transition $\mathrm{T}=68^{\circ} \mathrm{C}$, the interval between low and high bounds is much larger than at $\mathrm{T}<68^{\circ} \mathrm{C}$ and $\mathrm{T}>68^{\circ}$, thus, this large interval influences the fitness function and disperses slightly the estimated points. Even with those calculations errors, the results are in good agreement with experimental results presented in literature.

\section{IV.CONCLUSION}

In this work, we presented a numerical characterization of $\mathrm{VO}_{2}$; we succeeded the determination of the refractive index and the extinction coefficient using the particles swarm optimization algorithm and based on the theoretical model of Lorentz's oscillators. The results we obtained are similar to the experimental ones, we calculated the percentage of differences and found it around 7,5\% which is a good argument for the use of those results in further works about $\mathrm{VO}_{2}$ insulation based systems.

\section{REFERENCES}

[1] F.J. Morin. (1959) "Oxides which show metal-to-insulator transition at the neel temperature," Physical review letters (3), 34-36.

[2] J.B. Goodenough, "J. Solid state chem," Vol. 3, (1971), 490.

[3] Li, S. Y., Niklasson, G. A. \& Granqvist, C. G. "Thermochromic fenestration with VO2-based materials: Three challenges and how they can be met." Thin. Solid. Films 520, 3823-3828 (2012).

[4] G.V. Jorgensen, J.C. Lee, Solar Energy Mater. 14 (1986) 205.

[5] W. Burkhardt, T. Christmann, B.K. Meyer, W. Niessner, D. Schalch, A. Scharmann. (1999) "W- and F-doped VO2 films studied by photoelectron spectrometry,” Thin Solid Films 345, 229-235.

[6] K. Laaksonen, S.-Y. Li, S.R. Puisto, N.K.J. Rostedt, T. Ala-Nissila, C.G. Granqvist, R.M. Nieminen, G.A. Niklasson. (2014) "Nanoparticles of $\mathrm{TiO}_{2}$ and $\mathrm{VO}_{2}$ in dielectric media: Conditions for low optical scattering, and comparison between effective medium and four-flux theories," Solar Energy Materials and Solar cells vol. 130, 132-137. 
[7] Yanfeng Gao, Litao Kang, Zhang Chen and Hongjie Luo. (2011) "Solution processing of Nanoceramic $\mathrm{VO}_{2}$ Thin Films for Application to smart Windows, Nanofabrication," Dr. Yoshitake Masuda (Ed.), ISBN: 978-953-307-912-7, InTech, Available from: http://www.intechopen.com/books/nanofabrication/Solution-processing-of-nanoceramics-vo2-thin-films-for-application-to-smartwindows

[8] O. Iken, R. Agounoun, M. Rahmoune, K. Sbai, A. Zoubir, R. Saadani, A. Rahmani. (2017) "Numerical study of thermo-chromic properties of $\mathrm{VO}_{2}$ polymer composites coatings applied to building thermal insulation," International Journal of Engineering and Technology (IJET) vol. 9, No.2, 916-922.

[9] N.R. Mlyuka, G.A. Niklasson, C.G. Granqvist. (2009) "Thermochromic $\mathrm{VO}_{2}$-based multi-layer films with enhanced luminous transmittance and solar modulation," Phys. Status Solidi A, Vol. 206, 2155-2160.

[10] M. Clerc, J. Kennedy. (2002) "The Particle Swarm-Explosion, Stability, and Convergence in a Multidimentional Complex Space." IEEE Transactions on Evolutionary Computation, Vol. 6, No. 1, 58-73.

[11] J. McCall. (2005) "Genetic algorithms for modeling and optimization," Journal of Computational and Applied Mathematics 184, $205-$ 222.

[12] B. Nozohour-leilabady, B. Fazelabdolabadi. (2016) "On the application of artificial bee colony (ABC) algorithm for optimization of well placements in fractured reservoirs; efficiency comparison with the particle swarm optimization (PSO) methodology," Petroleum 2, 79-89.

[13] H. Kakiuchida, P. Jin, S. Nakao, M. Tazawa. (2007) "Optical Properties of Vanadium Dioxide Film during Semiconductive-Metallic Phase Transition,” Japanese Journal of Applied Physics vol. 46, No. 5, L113-L116.

[14] H. Kakiuchida, P. Jin, S. Nakao, M. Tazawa. (2008) "Optical characterization of Vanadium-titanium oxide Films," Thin solid films, $516,4563-4567$.

[15] I. F. Almog, M.S. Bradley, V. Bulovic. "The Lorentz Oscillator and its Applications," https://ocw.mit.edu/courses/electricalengineering-and-computer-science/6-007-electromagnetic-energy-from-motors-to-lasers-spring-2011/readings/ 